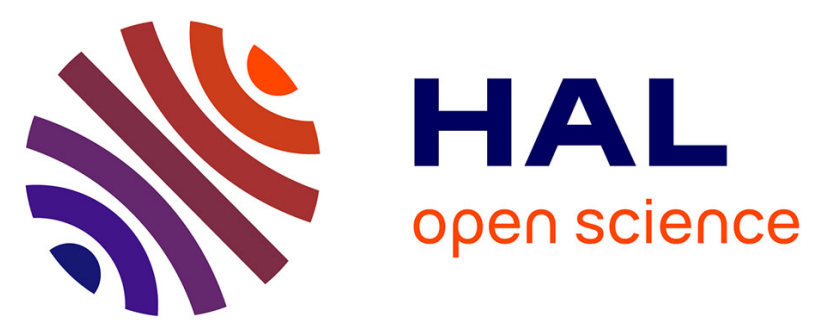

\title{
Formulation of Metal-Organic Framework Inks for the 3D Printing of Robust Microporous Solids toward High-Pressure Gas Storage and Separation
}

Jérémy Dhainaut, Mickaële Bonneau, Ryota Ueoka, Kazuyoshi Kanamori, Shuhei Furukawa

\section{To cite this version:}

Jérémy Dhainaut, Mickaële Bonneau, Ryota Ueoka, Kazuyoshi Kanamori, Shuhei Furukawa. Formulation of Metal-Organic Framework Inks for the 3D Printing of Robust Microporous Solids toward High-Pressure Gas Storage and Separation. ACS Applied Materials \& Interfaces, 2020, 12 (9), pp.10983 - 10992. 10.1021/acsami.9b22257 . hal-02998773

\section{HAL Id: hal-02998773 \\ https://hal.science/hal-02998773}

Submitted on 20 Nov 2020

HAL is a multi-disciplinary open access archive for the deposit and dissemination of scientific research documents, whether they are published or not. The documents may come from teaching and research institutions in France or abroad, or from public or private research centers.
L'archive ouverte pluridisciplinaire HAL, est destinée au dépôt et à la diffusion de documents scientifiques de niveau recherche, publiés ou non, émanant des établissements d'enseignement et de recherche français ou étrangers, des laboratoires publics ou privés. 


\section{ACS APPLIED MATERIALS \&INTERFACES}

Applications of Polymer, Composite, and Coating Materials

Subscriber access provided by GRIFFITH UNIVERSITY

Formulation of Metal-Organic Framework Inks for the 3D Printing of Robust Microporous Solids Towards High-Pressure Gas Storage and Separation

Jeremy Dhainaut, Mickaele Bonneau, Ryota Ueoka, Kazuyoshi Kanamori, and Shuhei Furukawa

ACS Appl. Mater. Interfaces, Just Accepted Manuscript • DOI: 10.1021/acsami.9b22257 • Publication Date (Web): 11 Feb 2020

Downloaded from pubs.acs.org on February 15, 2020

Just Accepted

"Just Accepted" manuscripts have been peer-reviewed and accepted for publication. They are posted online prior to technical editing, formatting for publication and author proofing. The American Chemical Society provides "Just Accepted" as a service to the research community to expedite the dissemination of scientific material as soon as possible after acceptance. "Just Accepted" manuscripts appear in full in PDF format accompanied by an HTML abstract. "Just Accepted" manuscripts have been fully peer reviewed, but should not be considered the official version of record. They are citable by the Digital Object Identifier (DOI®). "Just Accepted" is an optional service offered to authors. Therefore, the "Just Accepted" Web site may not include all articles that will be published in the journal. After a manuscript is technically edited and formatted, it will be removed from the "Just Accepted" Web site and published as an ASAP article. Note that technical editing may introduce minor changes to the manuscript text and/or graphics which could affect content, and all legal disclaimers and ethical guidelines that apply to the journal pertain. ACS cannot be held responsible for errors or consequences arising from the use of information contained in these "Just Accepted" manuscripts. 


\title{
1 Formulation of Metal-Organic Framework Inks for
} 2 the 3D Printing of Robust Microporous Solids 3 Towards High-Pressure Gas Storage and Separation

\author{
4 Jérémy Dhainaut ${ }^{a, t}{ }^{\dagger}$, , Mickaële Bonneau ${ }^{a}$, Ryota Ueoka ${ }^{b}$, Kazuyoshi Kanamori ${ }^{b}$, Shuhei
} 5 Furukawa $a^{a, c^{*}}$

6

7

8 a Institute for Integrated Cell-Material Sciences (WPI-iCeMS), Kyoto University, Yoshida, Sakyo9 ku, Kyoto 606-8501, Japan

$10{ }^{b}$ Department of Chemistry, Graduate School of Science, Kyoto University, Kitashirakawa, Sakyo-

11 ku, Kyoto 606-8502, Japan

$12{ }^{\mathrm{c}}$ Department of Synthetic Chemistry and Biological Chemistry, Graduate School of Engineering,

13 Kyoto University, Katsura, Nishikyo-ku, Kyoto 606-8510, Japan

14

15 Keywords: 3D Printing ; Metal-Organic Frameworks ; Inks Formulation ; Methane Storage ;

16 Ethane/Ethylene Separation 


\section{ABSTRACT.}

3 Shaping of metal-organic frameworks (MOFs) has become increasingly studied over the past few

4 years because it represents a major bottleneck toward their further applications at larger scale.

5 MOF-based macroscale solids should present similar performances to their powder counterparts

6 along with adequate mechanical resistance. 3D printing is one of the promising technologies as it

7 allows the fast prototyping of materials at the macroscale; however, the large amounts of added

8 binders have a detrimental effect on the porous properties of the solids. Herein, a 3D printer was

9 modified to prepare a variety of MOF-based solids with controlled morphology from shear-

10 thinning inks containing 2-hydroxyethyl cellulose. Four benchmark MOFs were tested for this

11 purpose: HKUST-1, CPL-1, ZIF-8 and UiO-66-NH 2 . All solids are mechanically stable up to 0.6

$12 \mathrm{MPa}$ of uniaxial compression and highly porous with BET specific surface areas lowered by 0 to

$13-25 \%$. Furthermore, these solids were applied to high pressure hydrocarbon sorption $\left(\mathrm{CH}_{4}, \mathrm{C}_{2} \mathrm{H}_{4}\right.$

14 and $\mathrm{C}_{2} \mathrm{H}_{6}$ ) and presented consequent methane gravimetric uptake (UiO-66-NH $\mathrm{NH}_{2}$ ZIF-8, and

15 HKUST-1) and highly preferential adsorption of ethylene of ethane (CPL-1). 


\section{INTRODUCTION}

Metal-organic frameworks (MOFs) constitute a fast-growing class of microporous

4 materials, assembled from metallic nodes - either ions or clusters - and multitopic organic linkers.

5 By changing the length, nature, and shape of linkers or the geometry of metallic nodes, one can

6 design the final pore size, shape and pore surface functionality. ${ }^{1,2}$ This exceptional structural

7 diversity strongly contributes to rank MOFs among the best materials reported so far in the

8 literature for catalysis and gas processing. ${ }^{3}$ Especially, in the context of the development of new

9 technologies for mobility, MOFs show some of the highest gravimetric capacities reported for

10 methane and hydrogen storage. ${ }^{4,5}$ As membranes, MOFs are also highly attractive for separation

11 in gas and liquid phases. ${ }^{6}$ Thus, a consequent effort has been deployed during the past few years

12 for rationalizing and scaling-up the synthesis of promising MOF structures using various

13 techniques such as continuous flow synthesis, ${ }^{7}$ spray-drying, ${ }^{8}$ mechanochemistry, ${ }^{9}$ or

14 electrochemistry. ${ }^{10}$ This dynamic led to the first commercial applications of MOFs in $2016 .{ }^{11}$

15 While MOFs are produced as loose powders and tested as such at the laboratory scale,

16 applications generally require handleable solids with a specific shape and sufficient mechanical

17 robustness to withstand overtime damaging stresses such as attrition and hydrostatic pressure. ${ }^{12}$

18 Therefore, various technologies are being evaluated for the shaping of MOFs. For example,

19 binderless pelletization proved to be a good compromise between mechanical stability and final

20 volumetric uptake in the cases of the zirconium-based $\mathrm{UiO}$ family and the $\mathrm{Cu}_{3}(\mathrm{btc})_{2}(\mathrm{btc}=1,3,5-$

21 benzenetricarboxylate) also known as HKUST-1. ${ }^{13}$ High gravimetric uptake was reported for

22 MOF-based grains prepared with an alumina binder, ${ }^{14}$ and with MOF beads prepared in the

23 presence of gelling agents. ${ }^{15}$ Multiporous monoliths could be obtained by gelation, freeze-casting,

24 or phase separation (spinodal decomposition) starting from a MOF dispersion, ${ }^{16-18}$ while well- 
1 controlled thin layers were obtained by chemical vapour deposition. ${ }^{19}$ Thus, most application

2 requirements can be covered owing to the current state-of-the-art of MOF shaping; however each

3 technique is limited to a narrow set of obtainable solids. Every application having different

4 specifications, and by anticipating that new applications will require unconventional shaping

5 methods, it is of tremendous interest to develop highly versatile techniques to produce robust

6 macroscale MOF solids.

7 Up to now, additive manufacturing, also called 3D printing, was mostly investigated to

8 produce highly dense ceramics and metal oxides with custom morphologies. ${ }^{20}$ Lately, a few

9 examples of porous solids were reported, including MOFs. MOF/thermoplastic composites were

10 prepared for direct use as replacements of the commercial polymer filaments in usual 3D

11 printers. ${ }^{21,22}$ When HKUST-1 crystals were mixed with an acrylate monomer and a photoinitiator,

12 porous and flexible macrostructures were obtained by a spatially-controlled polymerization step

13 (DLP). ${ }^{23}$ These methods are straightforward to implement on existing 3D printing setups, but the

14 resulting porous properties are heavily affected by the polymers' presence in significant quantity.

15 Instead of using filaments, another approach employs modified 3D printers to extrude inks

16 formulated in the presence of an organic binder. This robocasting approach was evaluated by $\mathrm{H}$.

17 Thakkar et al. to prepare MOF-74(Ni)- and UTSA-16(Co)-based materials with a woodpile

18 structure, ${ }^{24}$ which was also replicated by S. Sultan et al. starting from ZIF-8 and MIL-100(Fe), ${ }^{25}$

19 and A. J. Young et al. starting from UiO-66. ${ }^{26}$ In these cases, a fine control of the inks shear-

20 thinning properties is mandatory to obtain macroscale solids replicating the $3 \mathrm{D}$ models. These

21 materials presented an accessible porosity related to their MOF loading - between 30 and $85 \mathrm{wt} \%$

22 - and were successfully applied for the capture of guest molecules in gas- and liquid-phases. While 
1 these studies undeniably proved the advantages of combining inks and 3D printing, limited data

2 were given on the inks' rheology and the mechanical robustness of as-prepared solids.

This study focuses on the preparation of MOF-based solids by robocasting with controlled

4 macroscale morphology and superior textural properties. The general principle is illustrated on

5 Figure 1. In an effort to rationalize this approach, emphasis is given on measuring the viscosity of

6 inks formulated with four different MOF structures: $\left[\mathrm{Cu}_{2}(\mathrm{pzdc})_{2}(\mathrm{pyz})\right](\mathrm{CPL}-1$; pzdc $=$ pyrazine-

7 2,3-dicarboxylate, pyz $=$ pyrazine $),\left[\mathrm{Cu}_{3}(\mathrm{btc})_{2}\right](\mathrm{HKUST}-1 ;$ btc $=1,3,5$-benzenetricarboxylate $)$,

$8\left[\mathrm{Zn}(\mathrm{MeIm})_{2}\right](\mathrm{ZIF}-8, \mathrm{MeIm}=2$-methylimidazol-1-ide $)$ and $\left[\mathrm{Zr}_{6} \mathrm{O}_{4}(\mathrm{OH})_{4}(\text { bdc-NH})_{6}\right](\mathrm{UiO}-66-$

$9 \mathrm{NH}_{2}$, bdc- $\mathrm{NH}_{2}=2$-amino-1,4-benzenedicarboxylate). In a second step, the effect of the

10 robocasting process on the structural and porous properties of copper-paddlewheel-based HKUST-

11 1, which is reputed unstable, are investigated. Moreover, the mechanical robustness of as-prepared

12 solids is evaluated and compared to binderless pellets. Finally, these macroscale structures are

13 evaluated for high-pressure methane storage (HKUST-1, ZIF-8, UiO-66-NH ${ }_{2}$ ) and ethane/ethylene

14 separation (CPL-1), for which their powder counterparts present excellent performances.

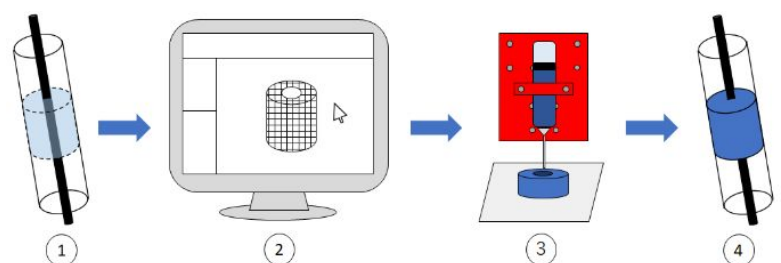

16 Figure 1. Typical process workflow based on the methodology developed herein: 1/ identification

17 of the need, 2/ computer-assisted modelling of the fitting solids, 3/ MOF-based ink deposition 18 using a 3D printer, 4/ evaluation of the performance of the solid.

\section{EXPERIMENTAL SECTION}


$2 N, N$-Dimethylformamide (DMF, $99.5 \%$ ), ethanol (EtOH, $99.5 \%$ ), methanol (MeOH, 99.8 \%),

3 Basolite Z1200 (ZIF-8), zirconium tetrachloride $\left(\mathrm{ZrCl}_{4}, \geq 97 \%\right)$, hydrochloric acid (HCl, $\left.35 \%\right)$,

4 2-aminoterephtalic acid $\left(\mathrm{H}_{2} \mathrm{BDC}-\mathrm{NH}_{2},>97 \%\right)$, copper acetate hydrate $\left(\mathrm{Cu}(\mathrm{OAc})_{2} .2 .5 \mathrm{H}_{2} \mathrm{O}, 99\right.$

$5 \%)$, copper nitrate hemi(pentahydrate) $\left(\mathrm{Cu}\left(\mathrm{NO}_{3}\right)_{2} \cdot 2.5 \mathrm{H}_{2} \mathrm{O},>98 \%\right)$, pyrazine-2,3-dicarboxylic

6 acid $\left(\mathrm{H}_{2} \mathrm{pzdc},>98 \%\right)$, pyrazine (pyz, > $\left.98 \%\right)$, trimesic acid $\left(\mathrm{H}_{3} \mathrm{BTC}, 95 \%\right)$, 2-hydroxyethyl

7 cellulose $(\mathrm{HEC}, \mathrm{Mw}=250000 \mathrm{~g} / \mathrm{mol})$, polyvinyl alcohol $(\mathrm{PVA}, \mathrm{Mw}=1500 \mathrm{~g} / \mathrm{mol})$. All reagents

8 and reactants were purchased from Wako Pure Chemical Industries or Sigma-Aldrich and were

9 used without further purification.

10 Synthesis of CPL-1 MOF. The synthesis is based on previous works from M. Bonneau et al. ${ }^{27} \mathrm{~A}$

11 solution of $\mathrm{Cu}\left(\mathrm{NO}_{3}\right)_{2} \cdot 2.5 \mathrm{H}_{2} \mathrm{O}(4.30 \mathrm{mmol})$ and pyz $(8.60 \mathrm{mmol})$ in $30 \mathrm{~mL}$ of $\mathrm{H}_{2} \mathrm{O}$ was stirred at

12 room temperature for 10 minutes. A solution of $\mathrm{H}_{2} \mathrm{pzdc}(4.30 \mathrm{mmol})$ with $\mathrm{NaOH}(8.52 \mathrm{mmol})$ in

13 a $30 \mathrm{~mL}$ mixture $7: 3$ of $\mathrm{H}_{2} \mathrm{O} / \mathrm{EtOH}$ was added dropwise to the stirring mixture. A blue precipitate

14 is noted. After one hour, the crude product is collected by centrifugation and was washed two times

15 with $\mathrm{H}_{2} \mathrm{O}$ and three times with EtOH. The blue powder is dried under vacuum at room temperature

16 overnight. Final yield $=95 \%$.

17 Synthesis of UiO-66-NH2 MOF. The synthesis is based on previous works from M.J. Katz et

$18 a .^{28}$ In a $110 \mathrm{~mL}$ glass vial, $2.08 \mathrm{mmol}$ of $\mathrm{ZrCl}_{4}$ were dissolved in a mixture of $20 \mathrm{~mL}$ of $\mathrm{DMF}$

19 and $4 \mathrm{~mL}$ of $\mathrm{HCl}$ under sonication for 20 minutes to obtain a clear solution. Then $2.92 \mathrm{mmol}$ of

$20 \mathrm{H}_{2} \mathrm{BDC}-\mathrm{NH}_{2}$ and $40 \mathrm{~mL}$ of DMF were added and the mixture was sonicated for an additional 20

21 min. The vial was left in static conditions in an oven at $80^{\circ} \mathrm{C}$. The product was recovered by

22 centrifugation, washed three times with $\mathrm{DMF}$, four times with $\mathrm{EtOH}$, and then dried overnight in

23 a vacuum oven at $100^{\circ} \mathrm{C}$. Final yield $=52 \%$. 
1 Synthesis of HKUST-1 MOF nanocrystals. The synthesis is based on previous works from J.

2 Huo et al. ${ }^{29}$ In a $1 \mathrm{~L}$ Erlenmeyer, $6.62 \mathrm{mmol}$ of $\mathrm{Cu}(\mathrm{OAc})_{2} .2 .5 \mathrm{H}_{2} \mathrm{O}$ were dissolved in $150 \mathrm{~mL}$ of 3 deionized $\mathrm{H}_{2} \mathrm{O}$. Meanwhile, $15.0 \mathrm{mmol}$ of $\mathrm{H}_{3} \mathrm{BTC}$ were dispersed in $150 \mathrm{~mL}$ of $\mathrm{H}_{2} \mathrm{O}$ under

4 sonication for $20 \mathrm{~min}$. The $\mathrm{H}_{3} \mathrm{BTC}$ suspension was quickly added to the $\mathrm{Cu}(\mathrm{OAc})_{2}$ solution and

5 the mixture was stirred at $1200 \mathrm{rpm}$ for $1 \mathrm{~h}$ at $23^{\circ} \mathrm{C}$. Additionally, $24 \mathrm{~h}$ in those conditions led to

6 the formation of larger crystals. The product was recovered by centrifugation, washed twice with

$7 \mathrm{MeOH}$, soaked overnight in $\mathrm{MeOH}$, and washed again twice with $\mathrm{MeOH}$. Finally, the powder was

8 dried overnight in a vacuum oven at $100^{\circ} \mathrm{C}$. Final yield $=29 \%$ (nanocrystals).

9 Preparation of shear-thinning inks. A stock solution of $\mathrm{HEC}$ in $\mathrm{H}_{2} \mathrm{O}$ was prepared by first 10 dissolving an adequate quantity of powder at $80^{\circ} \mathrm{C}$ under stirring and then leaving the obtained 11 clear solution in the fridge overnight before storage at room temperature. A defined quantity of

12 this stock solution was mixed at $1000 \mathrm{rpm}$ for $10 \mathrm{~h}$ with MOF powder deagglomerated beforehand 13 and PVA, here acting as a plasticizer during the subsequent printing step. For example, appropriate

14 shear-thinning properties were obtained with the following weight composition: 0.25 CPL-1 MOF:

150.01 PVA: 0.02 HEC: $0.65 \mathrm{H}_{2} \mathrm{O}$. Reproducibility of the protocol was verified by preparing three

16 times this ink' composition and measuring the relating apparent viscosity at different shear rates

17 (Figure S1). An average standard deviation of 16\% can be observed. Therefore, the following 18 apparent viscosities will be plotted as confidence intervals.

19 In the case of HKUST-1, a mixture $50 \mathrm{vol} \% \mathrm{H}_{2} \mathrm{O} / 50 \mathrm{vol} \% \mathrm{EtOH}$ is used instead of pure $\mathrm{H}_{2} \mathrm{O}$ to 20 limit hydrolysis.

21 Solids design and description of the robocasting process. The Part Design workbench of the 22 computer-assisted design software FreeCAD was used to model the different solids to be printed, 
1 then exported under a stereolithography (STL) file format. This file was loaded in Cura, a slicer

2 software, and relevant parameters for the 3D printing step were defined at this step. These

3 parameters include the printing speed, the temperature of the heating bed, and the thickness of the

4 layer deposited. The resulting set of data was saved under a g-code (GCODE) file, enabling to 5 pilot the 3D printer in a layer-by-layer fashion.

6 A modified Anet A8 3D printer, shown on Figure S2, was used for this work. According

7 to its technical specifications, its spatial resolution along $\mathrm{x} / \mathrm{y}$ axes is $\pm 0.2 \mathrm{~mm}$. The main

8 modifications include the addition of a borosilicate glass on the top of the heating bed for chemical

9 passivity and improved adhesion during ink deposition, and the replacement of the filament

10 feeding system by a customizable carriage printed with polylactic acid (PLA) to hold syringe

11 barrels filled with inks. The model was created by TNS and is licensed under the Creative

12 Commons - Attribution - Non-Commercial- Share Alike license on Thingiverse. The length of

13 the $0.8 \mathrm{~mm}$ diameter syringe needle was reduced to $20 \mathrm{~mm}$ to obtain a flat extremity and a constant

14 shear stress zone. Manual z-calibration was done between each experiment. To obtain a constant

15 flowrate during the printing, a peristaltic pump was pushing water upstream of the syringe, with a

16 rubber cap insuring no contact with the loaded ink and a homogeneous repartition of the pressure

17 applied. Accurate 3D replications were obtained with the following set of data: $20 \mathrm{~mm} / \mathrm{s}$ (printing

18 speed); $60{ }^{\circ} \mathrm{C}$ (heating plate temperature); $0.5 \mathrm{~mm}$ (layers thickness); $0.8 \mathrm{~mm}$ (needle diameter);

$197 \mu \mathrm{l} / \mathrm{s}$ (pump flow rate). As a simple accuracy test, a model made of lines and circles was printed

20 (Figure S3). When taking into account the spatial resolution of the printer, an average deviation of

$214 \%$ is observed between the model and the 3D printed solid. After partial drying on the heating 22 plate, the solids were recovered and dried overnight in a vacuum oven at $90^{\circ} \mathrm{C}$ before 23 characterization. 
1 Characterization and gas sorption. Viscosity of the inks at $23{ }^{\circ} \mathrm{C}$ was evaluated using a

2 Brookfield DV2T viscosimeter in a cone-plate configuration, with a cone radius of $2.4 \mathrm{~cm}$ and a 3 cone angle of $0.8^{\circ}$. Shear rates from 0.2 to $100 \mathrm{~s}^{-1}$ were applied in an increasing fashion, in a range

4 comprised between $10 \%$ and $100 \%$ of the maximum torque. Wide-angle powder X-ray diffraction 5 (PXRD) patterns were recorded on a Rigaku Ultima III diffractometer equipped with a $\mathrm{Cu} \mathrm{K}_{\alpha 1}$ 6 monochromatic radiation source $(45 \mathrm{kV}, 200 \mathrm{~mA})$. Diffraction patterns in the $5-50^{\circ}$ region were 7 recorded with a $0.02^{\circ}$ step size $($ step time $=0.3 \mathrm{~s})$. Before measurement, the powder was deposited 8 on a glass substrate and automatic alignment was made. Thermogravimetry-differential thermal 9 analysis (TG-DTA) was performed on Rigaku Thermo plus EVO II between 23 and $800{ }^{\circ} \mathrm{C}$, at a 10 heating rate of $5^{\circ} \mathrm{C} \cdot \mathrm{min}^{-1}$ under nitrogen flow. Microstructures of the as-prepared MOF crystals 11 and the fractured surfaces of robocasted solids were observed under a Hitachi SU5000 scanning 12 electron microscope (SEM). Samples were directly deposited on a conductive carbon tape and 13 coated with $10 \mathrm{~nm}$ of osmium. Fourier-Transform Infrared (FT-IR) spectra were recorded in 14 attenuated total reflectance (ATR) using a Jasco FT/IR-6100 spectrometer equipped with a 15 TriGlycine Sulfate detector.

16 Mechanical properties of 3D-printed solids were compared to their binderless pelletized 17 counterparts, prepared using a AS ONE manual tableting instrument. The die, with an inner 18 diameter of $10 \mathrm{~mm}$, was filled with an appropriate amount of MOF powder to obtain a final height 19 of $5 \mathrm{~mm}+/-0.5 \mathrm{~mm}$ after maintaining a pressure of 200 bars for 2 minutes. The unidirectional 20 compression tests were carried out using a material tester (Autograph AG-X plus, Shimadzu Corp., 21 Japan). The pressure applied on the force sensor was recorded as a function of the displacement of 22 the punch at a constant speed of $5.0 \mathrm{~mm} \cdot \mathrm{min}^{-1}$. One representative solid of each composition and 23 from each shaping technique was tested. 
Prior to sorption isotherm measurements, all samples were degassed at $120{ }^{\circ} \mathrm{C}$ under

2 secondary vacuum during $14 \mathrm{~h}$. Nitrogen $\left(\mathrm{N}_{2}\right)$ and carbon dioxide $\left(\mathrm{CO}_{2}\right)$ adsorption isotherms were

3 recorded on a BEL Japan BELSORP-mini volumetric-adsorption instrument at $77 \mathrm{~K}$ and $195 \mathrm{~K}$,

4 respectively. Specific surface areas (SSA) were determined using the BET method on a linear

5 portion of the nitrogen adsorption isotherm below $\mathrm{P} / \mathrm{P}^{0}=0.3$, with $\mathrm{R}>0.999$ and $\mathrm{C}>0$. The

6 micropore $\left(\mathrm{V}_{\text {micro. }}\right)$ and total pore $\left(\mathrm{V}_{\text {tot. }}\right)$ volumes were estimated from the adsorbed volume of $\mathrm{N}_{2}$

7 at $\mathrm{P} / \mathrm{P}^{0}=0.30$ and 0.99 , respectively. High pressure methane $\left(\mathrm{CH}_{4}\right)$, ethane $\left(\mathrm{C}_{2} \mathrm{H}_{6}\right)$ and ethylene

$8\left(\mathrm{C}_{2} \mathrm{H}_{4}\right)$ sorption isotherms were recorded on a BEL Japan BELSORP-max volumetric-adsorption

9 instrument at $298 \mathrm{~K}$ up to 55 bars $\left(\mathrm{CH}_{4}\right)$ and 19 bars $\left(\mathrm{C}_{2} \mathrm{H}_{4}, \mathrm{C}_{2} \mathrm{H}_{6}\right)$. Expected deviation on

10 physisorption measurements is around $\pm 1 \%$.

\section{RESULTS AND DISCUSSION}

\section{Optimization of MOF inks' composition}

13 At first, the composition of inks containing MOF crystals was optimized to obtain the best

14 compromise between high loading of MOF crystals and good processability. For robocasting, inks

15 should demonstrate controlled shear-thinning properties, with proper flowability when an external

16 force is applied, while holding their shape in stationary state. Thus, it is expected that the inks

17 should be as viscous as possible, within a range allowing a continuous flow rate by the peristaltic

18 pump. This flow rate also has to be in adequation with the printing speed of the $3 \mathrm{D}$ printer and the

19 thickness of the deposited layer. Because many parameters have a notable influence on the printing

20 quality, all parameters relating to the 3D-printing operation were set constant and only the inks

21 composition was modified accordingly. After a preliminary screening, a combination of 2-

22 hydroxyethyl cellulose (HEC) and polyvinyl alcohol (PVA) was selected as binders over Pluronic 
1 F-127 and polyvinylpyrrolidone K25 owing to the higher viscosity obtained using small $(<10$

$2 \mathrm{wt} \%$ ) quantities. This is mandatory to maximize the porosity of the final object and hence its

3 performances. HEC is derived from cellulose and is widely used as a gelling agent in cosmetics

4 and household products. Note that the use of methylcellulose, another product derived from

5 cellulose, was reported for the preparation of zeolite-based monoliths by 3D-printing. ${ }^{30}$

$6 \quad$ Figure 2 presents the apparent viscosity of inks formulated with different concentrations of

7 CPL-1, while maintaining constant MOF/HEC and HEC/PVA ratio. All points can be fitted by a

8 power law with a negative exponent, which is characteristic of a shear-thinning behaviour. ${ }^{31}$

9 Higher concentrations lead to higher apparent viscosities, with more than a two-fold increase of

10 the viscosity when the concentration is increased by a factor 1.5 . When used for robocasting, solids

11 replicating the models were successfully obtained with the ink formulated with $35 \mathrm{wt} \%$ of CPL-1

12 (Figure 3a). On the other hand, poorly defined solids were obtained with the more concentrated 13 ink (51 wt \%), as evidenced on Figure 3b.

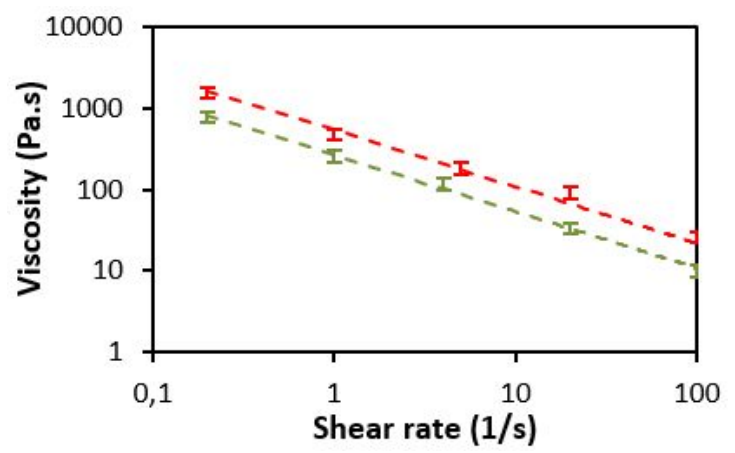

15 Figure 2. Viscosity plots of inks formulated with $35 \mathrm{wt} \%$ (green) and $51 \mathrm{wt} \%$ (red) of CPL-1.

$16 \mathrm{MOF} / \mathrm{HEC}=12.5 ; \mathrm{HEC} / \mathrm{PVA}=2.0$. Error bars $=10 \%$.

17 A similar observation was made with inks formulated with different concentrations of ZIF188 (Figure S4). This is because of limitations induced by the use of a peristaltic pump, whose torque 
1 is not able to maintain a constant flow rate of inks with apparent viscosities above $1000 \mathrm{~Pa}$.s under

2 a shear rate of $0.2 \mathrm{~s}^{-1}$. Therefore, this value was set as a higher limit when formulating MOF-based

3 inks. On the other hand, when the apparent viscosity at low shear rate is ten-fold lower, consequent

4 spreading of the ink occurs after its deposition on the plate (Figure S5), resulting in a poor 5 replication of the 3D model. Therefore, a careful balance needs to be reached.

6

7 Figure 3. Photographs of CPL-1-based objects printed under the same conditions with inks

8 formulated with $35 \mathrm{wt} \%$ (a) and $51 \mathrm{wt} \%$ (b) of CPL-1.

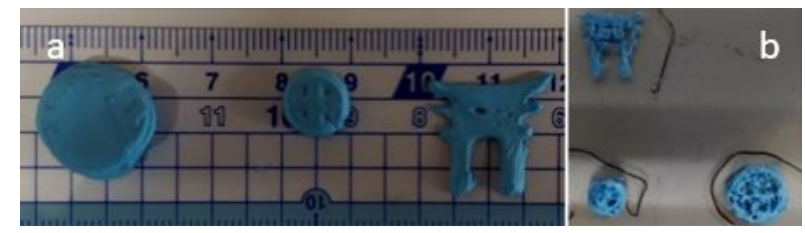


The highest concentration of crystals for a processable ink was obtained in the presence of

2 CPL-1 (35 wt\%). CPL-1 consists of 2D layers of copper(II) and pyrazine, pillared by pyrazine3 2,3-dicarboxylate compounds. The resulting crystals are highly anisotropic, as displayed in Figure 4 S7. In comparison, the crystals obtained with HKUST-1, ZIF-8, and UiO-66- $\mathrm{NH}_{2}$ are isotropic 5 (Figures S6 and S7). The influence of anisotropic particles, and especially clays, on the rheological 6 properties of suspensions has been extensively studied. ${ }^{34}$ In particular, under high shear rates, 7 anisotropic materials becomes oriented with the flow direction, therefore minimizing the intrinsic 8 viscosity and increasing the maximum packing density. Interestingly, this observation further 9 means that anisotropic materials might be more suited for the 3D printing of inks.

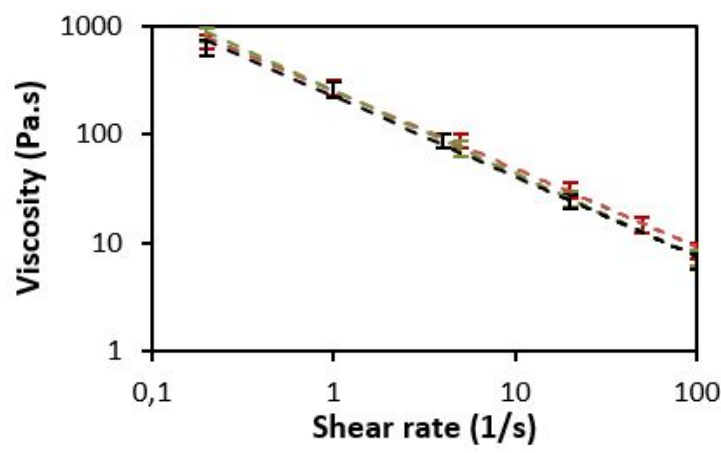

11 Figure 4. Viscosity plots of a pure HEC solution (black), and HKUST-1-based inks formulated 12 with $11 \mathrm{wt} \%$ of large crystals (red) and with $16 \mathrm{wt} \%$ of nanocrystals (green). MOF/HEC $=4.9$ 13 (blue) and 6.9 (green); HEC/PVA = 2.1.

14 Therefore, viscosity is proposed as a reliable descriptor to evaluate the inks processability, 15 with apparent viscosities comprised between 100 and 1000 Pa.s under a shear rate of $0.2 \mathrm{~s}^{-1}$ and a 16 shear-thinning behaviour allowing their use for robocasting. These criteria should be met in future 17 studies involving MOF-based inks and similar setups.

\section{Characterization of the 3D printed solids}


1 Fresh inks formulated with the different MOF structures were prepared and their measured

2 apparent viscosity is given on Figure S8. Cylinders of $10 \times 10 \mathrm{~mm}$ were prepared for

3 characterization and mechanical tests, along with various solids displayed on Figure S9. After

4 drying, these solids present a uniform volume shrinkage of about 6\%. (Figure S10).
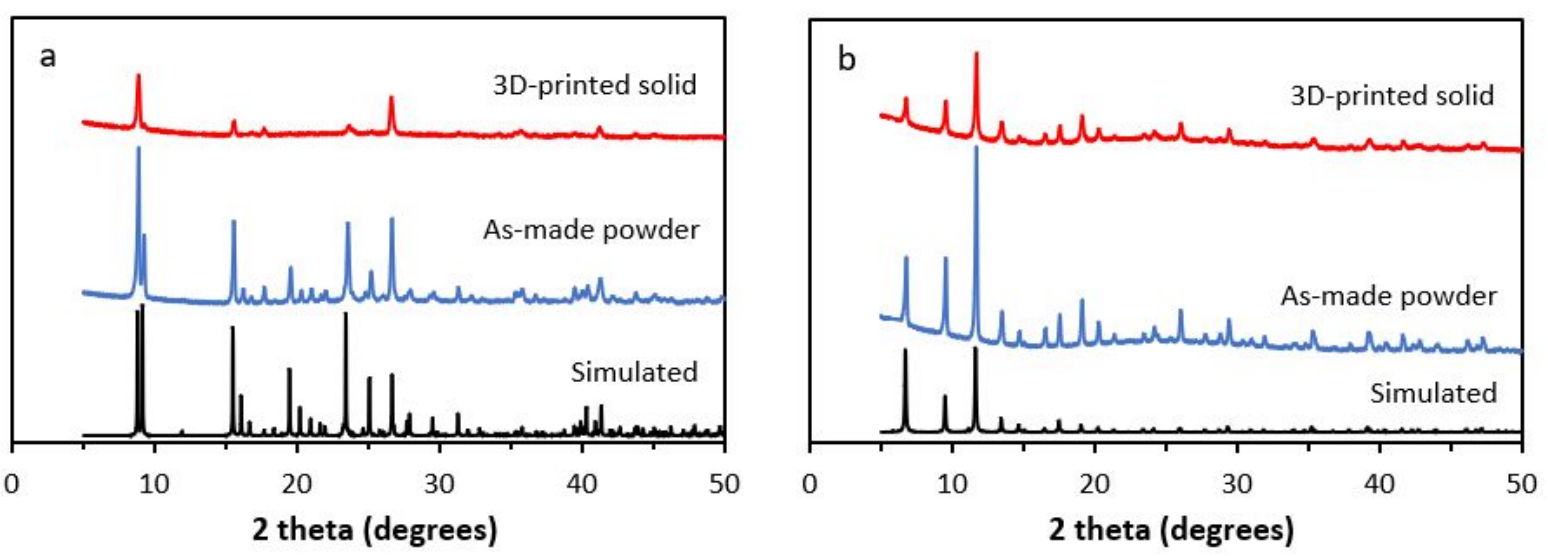

5
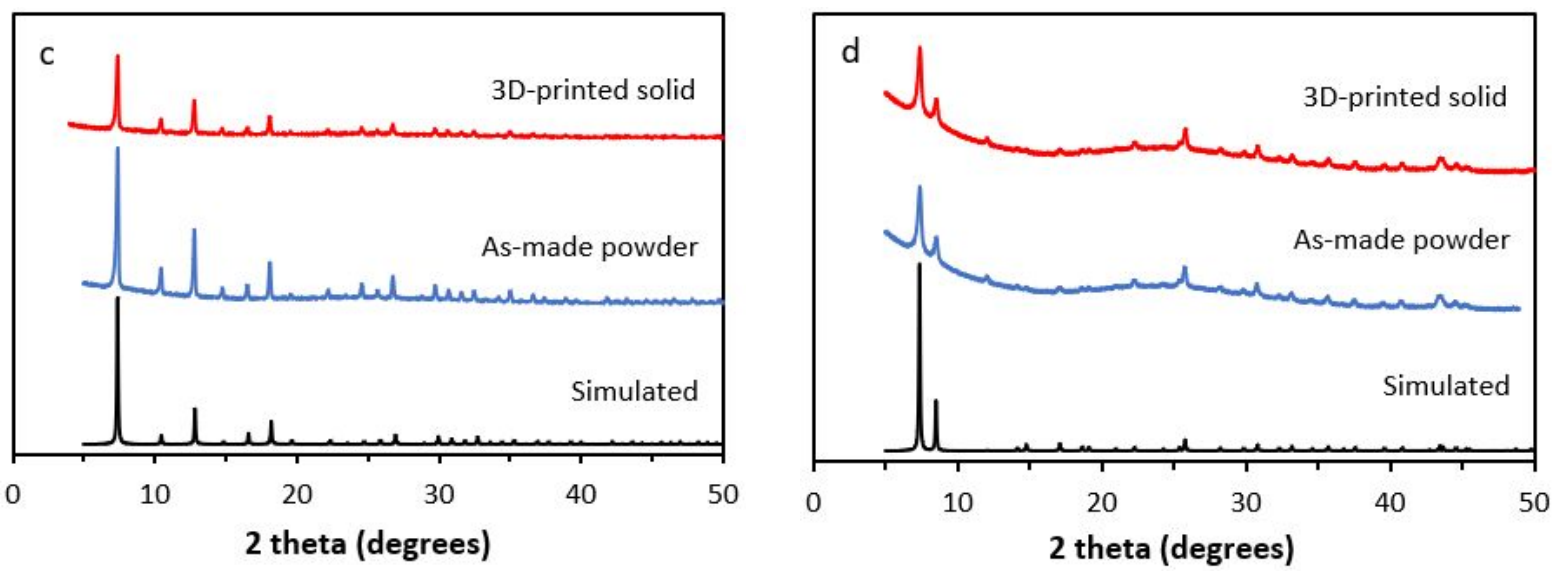

7 Figure 5. PXRD patterns of the as-made MOF powders and the related 3D-printed solids: CPL-1

8 (a), HKUST-1 (b), ZIF-8 (c) and UiO-66- $\mathrm{NH}_{2}$ (d).

$9 \quad$ Figure 5 presents PXRD patterns of the as-prepared MOF powders and the 3D-printed 10 solids. As evidenced by the diffraction data, the robocasting process only slightly affected the 11 structures of MOFs. No secondary crystalline phase can be detected, while lower diffraction 
1 intensities can be attributed either to a partial loss of long-range order - especially in the case of

2 HKUST-1 - or to the presence of amorphous PVA and HEC in significant quantity. Their presence

3 is also attested by thermogravimetric analysis (TGA) under $\mathrm{N}_{2}$ flow (Figure S11), with an

4 increased weight loss above $200{ }^{\circ} \mathrm{C}$ matching with the ink's composition. In the case of CPL-1,

5 the 3D-printed solid presents different relative intensities compared to the as-made powder

6 (Figures 5a). This is due to the preferential orientation of the anisotropic crystals along the $b$-axis

7 after formulation of the ink and deposition, as observed on Figure S12. Infrared spectroscopy with

8 attenuated total reflection mode (IR-ATR) was also conducted on all samples and revealed similar

9 surface properties between the as-prepared powders and the 3D-printed solids (Figure S13). An

10 additional low-to-medium band at $1060 \mathrm{~cm}^{-1}$ can be attributed to primary alcohol functions coming

11 from the hydroxyethyl cellulose in the 3D-printed solids. Thus, the 3D-printed solids retained the

12 MOF microporous structures even in the case of the more sensitive copper-paddlewheel-based

13 structures.

14 Porous properties of as-made powders and 3D-printed solids were confronted using $\mathrm{CO}_{2}$

15 and $\mathrm{N}_{2}$ physisorption, and the resulting isotherms are presented on Figure 6 and Figure S14,

16 respectively. For direct comparison, the adsorbed volume was recalculated using the proportion of

17 MOF in the solids as indicated by TGA (Tab. S1). In the case of ZIF-8, comparable isotherms are

18 obtained with both gases ( -1 to $+3 \%)$. This means that neither the binders' presence or the shaping

19 process affect the adsorption capacity of the ZIF- $8 \mathrm{MOF}$, and by extension that the binders should

20 not modify significantly the adsorption capacity of any MOF. 

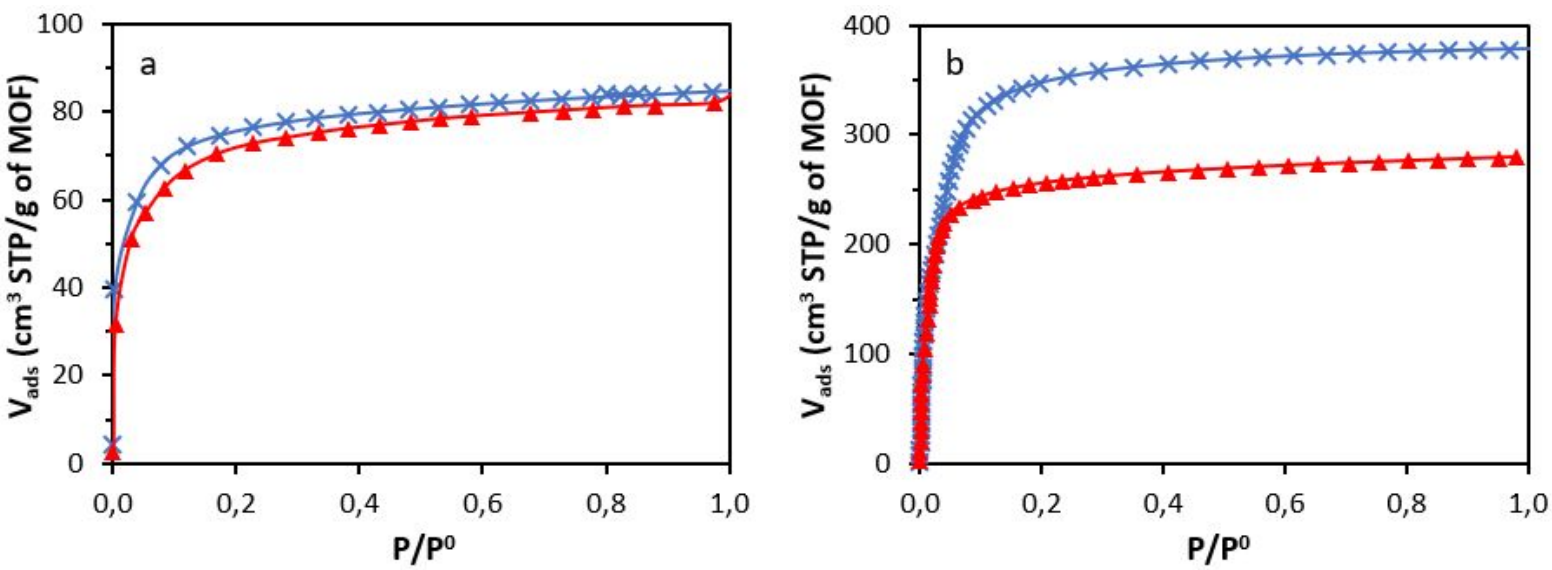

1
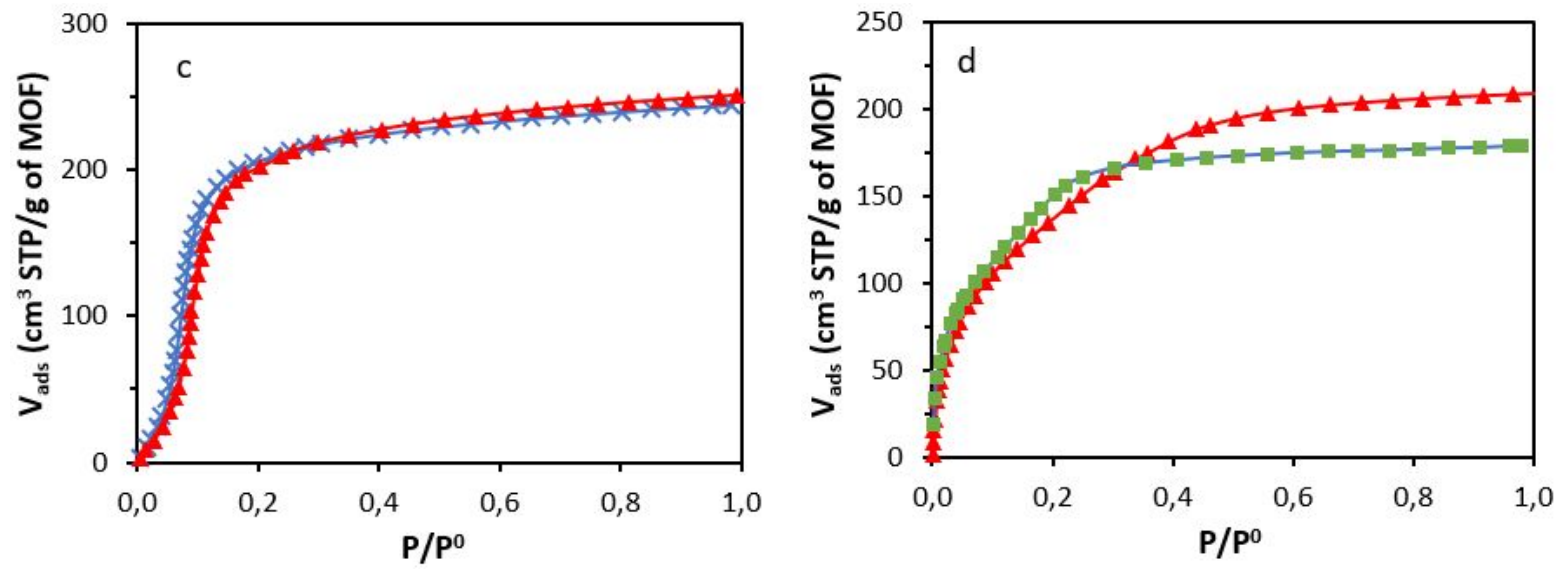

3 Figure 6. Carbon dioxide adsorption isotherms at $195 \mathrm{~K}$ of the as-made MOF powders (cross), 4 and the related crushed pellets (square) and 3D-printed solids (triangle): CPL-1 (a), HKUST-1 (b), $5 \quad \mathrm{ZIF}-8$ (c) and UiO-66- $\mathrm{NH}_{2}$ (d).

After 3D-printing UiO-66- $\mathrm{NH}_{2}$ also presents a comparable type I $\mathrm{N}_{2}$ adsorption isotherm

7 (Figure S14), with a comparable specific surface area. This is expected as $\mathrm{UiO}-66-\mathrm{NH}_{2}$ was

8 reported as highly stable - except in basic conditions. ${ }^{35}$ More interestingly, a significant increase

9 of about $+17 \%$ is observed on the total adsorbed $\mathrm{CO}_{2}$ isotherm after 3D-printing, compared to a

10 crushed pellet of the same as-made powder. Using a crushed pellet of UiO-66- $\mathrm{NH}_{2}$ instead of the

11 as-made powder should result in a difference of less than 5\%, owing to the resistance of this 
1 framework towards uniaxial compression. ${ }^{13}$ Under a relative pressure of 0.25 , both isotherms

2 follow the same adsorption trend, with the adsorption branch after 3D-printing being below the

3 branch of the crushed pellet. This can be interpreted as a small loss of pore accessibility due to the

4 presence of binders. However, above a relative pressure of 0.25 , the porosity is saturated in the

5 case of the crushed pellet while the adsorbed volume continues to increase as a function of the

6 relative pressure in the case of the 3D-printed solid. Owing to the small size of the crystals $(<150$

$7 \mathrm{~nm}$, Figure S7), and the increased adsorption capacity at higher relative pressure, this observation

8 is most likely correlated to the formation of interstitial voids between crystals due to the presence

9 of binders.

10 After shaping, HKUST-1 presents a lowered BET specific surface area of about $-25 \%$ 11 along with a decreased $\mathrm{CO}_{2}$ adsorption capacity $(-30 \%)$. This is in line with the XRD

12 characterization and indicates that HKUST-1 was partially degraded after the 3D-printing process.

13 This MOF structure is reputed to undergo hydrolysis in the presence of water. ${ }^{36}$ To avoid this 14 partial degradation, water should be completely replaced by another polar solvent that can dissolve 15 HEC, such as dichloroethane. Comparatively, other shaping techniques such as pelletization 16 commonly lowers HKUST-1 specific surface area by -15 to $-95 \%$, depending on the pressure 17 applied. ${ }^{13}$

Another result of interest comes from the CPL-1 3D-printed solid. While this solid shows

19 similar $\mathrm{CO}_{2}$ adsorption capacity at $195 \mathrm{~K}$ compared to its powder counterpart, a greatly reduced

$20 \mathrm{~N}_{2}$ adsorption capacity is observed at $77 \mathrm{~K}$ (-73\% micropore volume). Regular $\mathrm{CO}_{2}$ sorption is

21 observed following the $\mathrm{N}_{2}$ sorption of the same solid. Knowing that some flexibility was reported

22 with these pillared-layer structures, ${ }^{37}$ this observation could be related to a combined effect of 
1 framework flexibility and a different behaviour from HEC and PVA at $77 \mathrm{~K}$, inducing a partial 2 pore blockage.
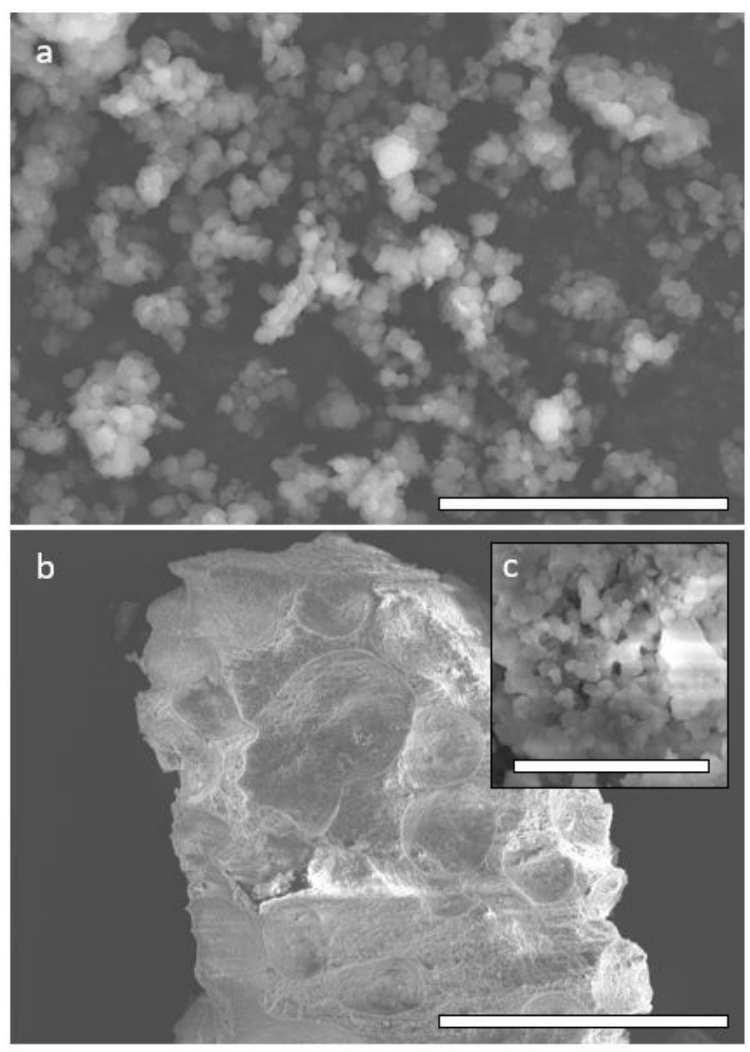

4 Figure 7. SEM micrographs of as-made ZIF-8 powders (a), and a fragment of 3D-printed ZIF-8

5 solid (b, c). Scale bars are $10 \mu \mathrm{m}(\mathrm{a}, \mathrm{c})$ and $500 \mu \mathrm{m}(\mathrm{b})$.

6 SEM micrographs of ZIF-8 are presented on Figure 7. No difference can be observed in 7 terms of size and morphology between the as-prepared powder and the 3D-printed solids.

8 Interestingly, craters can be observed on 3D-printed solid fragments, resulting from the fast drying

9 of inks after printing. While water represents more than $50 \mathrm{vol} \%$ of the inks' composition, after

10 drying no modification of the solids dimensions is observed. Therefore, a consequent volume

11 fraction of the solids must be empty. A plausible explanation comes from the fact that, owing to

12 the shear-thinning properties of the inks, the solvent has higher mobility than the suspended 
1 crystals, resulting in convective heat transfer being faster than conductive heat transfer (Peclet

2 number $>1$ ) and hence in local radial diffusion of the crystals. ${ }^{38}$ This phenomenon is similar to the

3 formation of hollow spheres by spray-drying. ${ }^{7}$

4 As a partial conclusion, 3D-printed solids were obtained from CPL-1, HKUST-1, ZIF-8

5 and $\mathrm{UiO}-66-\mathrm{NH}_{2}$ as-prepared powders with low impact on their structural and porous properties

6 (0 to -25\%). In comparison, H. Thakkar et al. observed a decrease of the recalculated micropore

7 volume comprised between $-7 \%(\mathrm{USTA}-16(\mathrm{Co}))$ and $-22 \%(\mathrm{MOF}-74(\mathrm{Ni}))$ in the presence of

8 bentonite and PVA, ${ }^{24}$ and S. Sultan et al. observed a decrease of $-27 \%$ of the micropore volume

9 of ZIF-8 in the presence of modified cellulose nanofibers and alginate. ${ }^{25}$ This difference is due to

10 the use of HEC and PVA in low amounts (7-20 wt \%), allowing high accessibility of the inner

11 porosity. Therefore, the inks' preparation and the robocasting methodologies described here are

12 adapted for the preparation of 3D-printed MOF-based solids with the original porous properties

13 maintained.

\section{Evaluation of their mechanical stability}
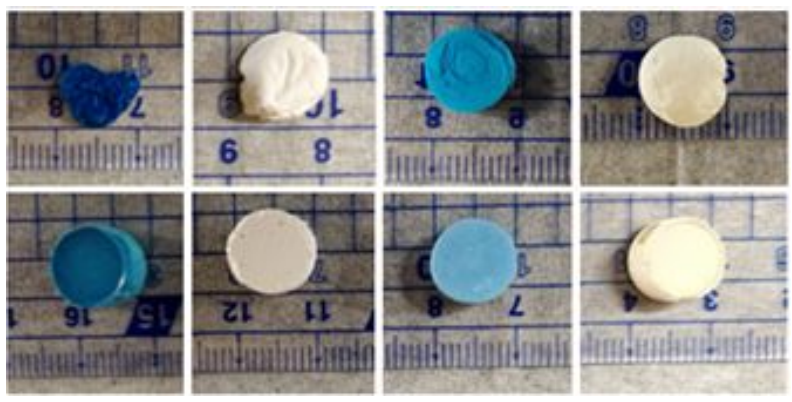

16 Figure 8. Photographs of the cylindrical 3D-printed solids (top) and their binderless pelletized

17 counterparts (bottom), whose mechanical resistance was evaluated by uniaxial compression. From

18 left to right: HKUST-1, ZIF-8, CPL-1, and UiO-66-NH2. 
1 For long term application and cyclability, it is necessary to evaluate the mechanical

2 robustness of highly porous solids. Insufficient mechanical stability leads to their attrition,

3 generation of dust, and therefore, overtime loss of performances. In continuous flow application

4 such as gas separation, the solids also have to withstand the applied pressure drop.

5 The mechanical properties of 3D-printed solids were evaluated by uniaxial compression.

6 Cylinders of dimensions $10 \times 10 \mathrm{~mm}$ were pressed on their flat surface at a constant displacement

7 rate and the pressure was recorded until sudden pressure drop. For comparison, binderless tablets

8 of identical dimensions were also prepared using a manual tableting instrument. All these solids

9 are presented on Figure 8. As the CPL-1-based ink contained the highest MOF crystals loading,

10 almost perfectly shaped cylindrical solids were prepared. On the other hand, lower MOF crystals

11 loadings induced partial sagging on the edges, which is expected to have an effect on the

12 mechanical test by lowering the surface in contact with the die and thus underestimating their

13 compressive strength.
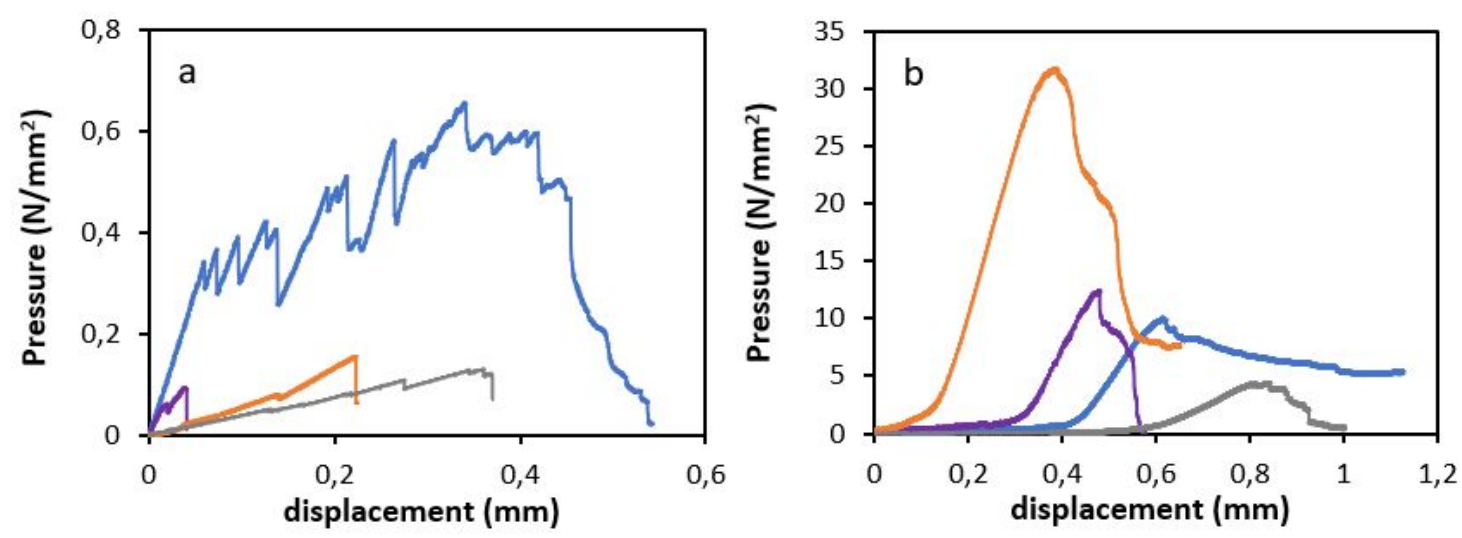

15 Figure 9. Compressive pressure-displacement curves of cylindric 3D-printed solids (a) and 16 binderless tablets (b): CPL-1 (blue), HKUST-1 (purple), ZIF-8 (grey), and UiO-66-NH2 (orange). 
1 The compressive pressure-displacement curves of all tested solids are presented in Figure

2 9. One to two orders of magnitude of compressive strength difference can be observed between

3 the 3D-printed samples and the binderless tablets, defined as the maximum pressure recorded

4 before catastrophic failure. This can be explained by the considerable (>50 vol\%) voids fraction

5 inside the 3D-printed solids, favouring the fracture propagation. Nonetheless, these results are

6 remarkable as the CPL-1 solid 3D-printed from an ink incorporating $35 \mathrm{wt} \%$ of MOF crystals

7 presents compressive strength $(0.64 \mathrm{MPa})$ in line with the solids prepared by $\mathrm{H}$. Thakkar et al.

$8(0.56 \mathrm{MPa}),{ }^{24}$ while incorporating less than $7 \mathrm{wt} \%$ of binders. This is highly desirable in order to 9 maximize the storage capacity of the materials.

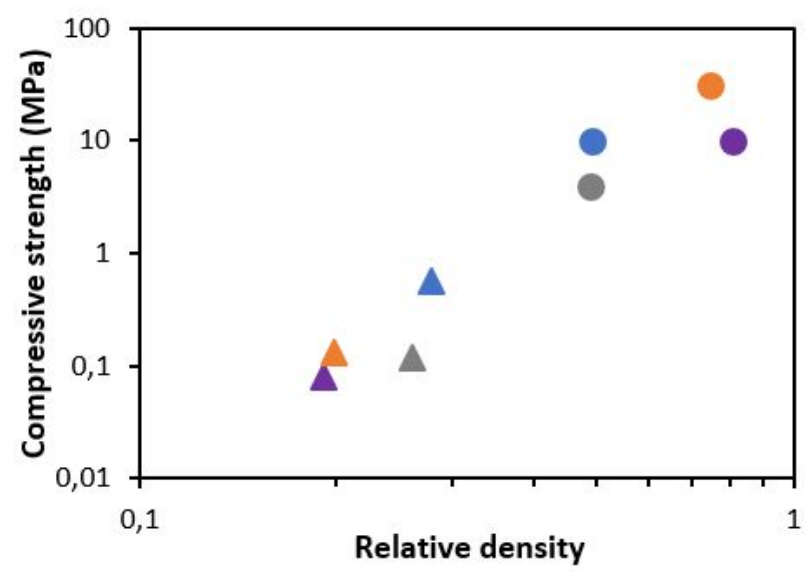

11 Figure 10. Compressive strength as a function of the relative density of 3D-printed solids (triangle)

12 and binderless pellets (circles) prepared from CPL-1 (blue), HKUST-1 (purple), ZIF-8 (grey), and 13 UiO-66- $\mathrm{NH}_{2}$ (orange) as-made powders.

14 To investigate more in details the effect of the voids fraction on the compressive strength 15 of the materials, Figure 10 presents the latter as a function of their relative density. A correlation 16 can be seen, unrelated to the shaping technique used. This correlation was also reported in the case 17 of macroporous ceramics. ${ }^{39}$ Hence, the most straightforward way to further increase the 
1 mechanical performances of 3D-printed solids is to increase their relative density. As previously

2 underlined, this increase can be obtained by decreasing the applied drying temperature in order to

3 slow down convective heat transfer. The resulting solids' dimensions will then shrink during

4 drying, leading to denser crystals' packing.

\section{Use of 3D-printed monoliths for gas storage and separation}

6 MOFs are actively screened for gas storage owing to their superior porous properties.

7 Notably, methane is seeing as an alternative to petrol for mobility applications, and MOFs show

8 some of the highest volumetric and gravimetric capacities reported so far, with HKUST-1 being

9 one of the leading materials. ${ }^{4}$ However, most studies evaluated methane storage capacity of MOFs

10 in powder form, ${ }^{40,41}$ while mobility applications require well-defined, attrition-resistant solids.

11 Therefore, high pressure methane adsorption/desorption isotherms were measured at ambient

12 temperature on the 3D-printed cylinders up to 55 bars, and displayed on Figure 11.

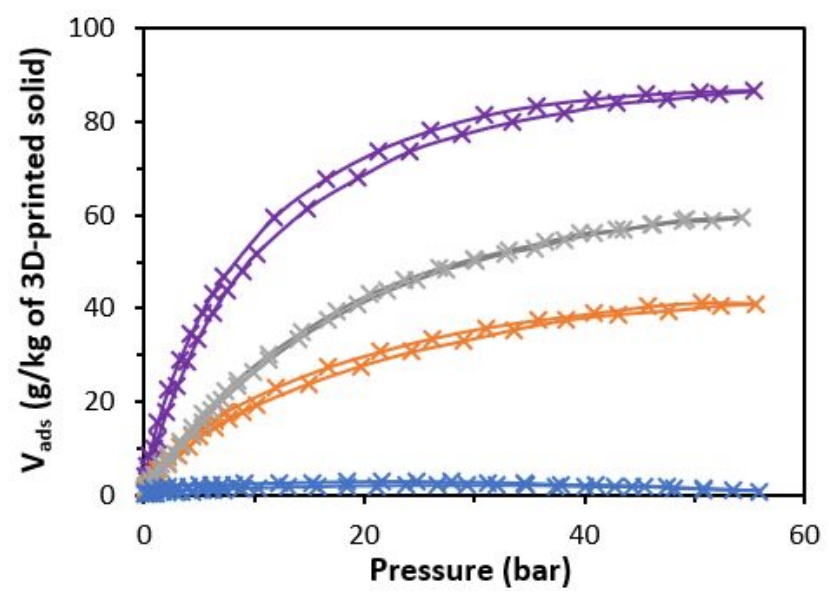

14 Figure 11. High-pressure methane physisorption isotherms at $298 \mathrm{~K}$ of 3D-printed monoliths:

15 CPL-1 (blue), HKUST-1 (purple), ZIF-8 (grey) and UiO-66- $\mathrm{NH}_{2}$ (orange). 
In the literature a simple correlation between the gravimetric uptake of methane and the

2 BET specific surface area of MOFs was proposed, with a plateau reached above $2400 \mathrm{~m}^{2} / \mathrm{g} .{ }^{40}$

3 According to this correlation, our HKUST-1 3D-printed solids with a BET specific surface area of

4 about $1200 \mathrm{~m}^{2} / \mathrm{g}$ should present a total gravimetric uptake of about $105 \mathrm{~g}$ of methane per kilogram

5 of MOF at $298 \mathrm{~K}$ and 35 bar. This value is in accordance with the $81 \mathrm{~g}$ of methane adsorbed per

6 kilogram of 3D-printed HKUST-1-based solid at $298 \mathrm{~K}$ and 35 bar. After taking into account the

$719.5 \mathrm{wt} \%$ of HEC and PVA content, the total gravimetric uptake reaches $101 \mathrm{~g}$ of methane

8 adsorbed per kilogram of HKUST-1. Similar conclusion is drawn with the ZIF-8 3D-printed solids,

9 presenting a gravimetric capacity of $59 \mathrm{~g}$ of methane per kilogram of ZIF-8 at $298 \mathrm{~K}$ and 30 bar

10 (against a reported $68 \mathrm{~g} / \mathrm{kg}$ of commercial ZIF-8 powder at $298 \mathrm{~K}$ and $30 \mathrm{bar}$ ). ${ }^{42}$

11 UiO-66- $\mathrm{NH}_{2}$ presents the highest gravimetric methane uptake of all isoreticular UiO-66

12 frameworks (76 g/kg of MOF at $298 \mathrm{~K}$ and 20 bar), owing to its small and polar amino groups. ${ }^{43}$

13 Herein, the UiO-66- $\mathrm{NH}_{2}$-based 3D-printed solids display lower methane uptake $(30 \mathrm{~g} / \mathrm{kg}$ of MOF

14 at $298 \mathrm{~K}$ and 20 bar), which could be due to its significantly lower BET specific surface area (871

$15 \mathrm{~m}^{2} / \mathrm{g}$ instead of $1123 \mathrm{~m}^{2} / \mathrm{g}$ ). It seems that the presence of HEC and PVA does not affect

16 significantly the adsorption performances of the MOFs for methane storage, as also observed for

$17 \mathrm{~N}_{2}$ and $\mathrm{CO}_{2}$ adsorption. After testing the solids were recovered with no visible failure and are

18 therefore stable up to 55 bars. Thus, 3D printing could allow to design solids specific for mobility

19 applications, easily stackable and with the controlled formation of macro-holes for heat dissipation

20 and constant methane delivery. Further effort should focus on obtaining highly porous 3D-printed

21 solids, with BET specific surface areas above $2000 \mathrm{~m}^{2} / \mathrm{g}$.

22 While CPL-1 shows neglectable methane adsorption capacity compared to other MOFs

23 (Figure 11), this framework presents one of the highest reported separation capacities for C2 
1 hydrocarbon mixtures owing to its small one-dimensional channels (4 $\AA$ x $6 \AA$ ) and flexible

2 behaviour, with the complete exclusion of ethane over ethylene at room temperature and mild

3 pressure. ${ }^{44}$ By comparison with the ethane gas molecule, the relatively smaller size of ethylene

4 (less than $0.2 \AA$ difference) and the interaction of ethylene double-bond with the open metal site

5 allow the adsorption of ethylene at mild pressure. ${ }^{45,46}$ Only a few materials are presenting this

6 behavior which is highly interesting for gas purification ${ }^{47}$ generally relying on highly energy-

7 costly cryogenic distillation.

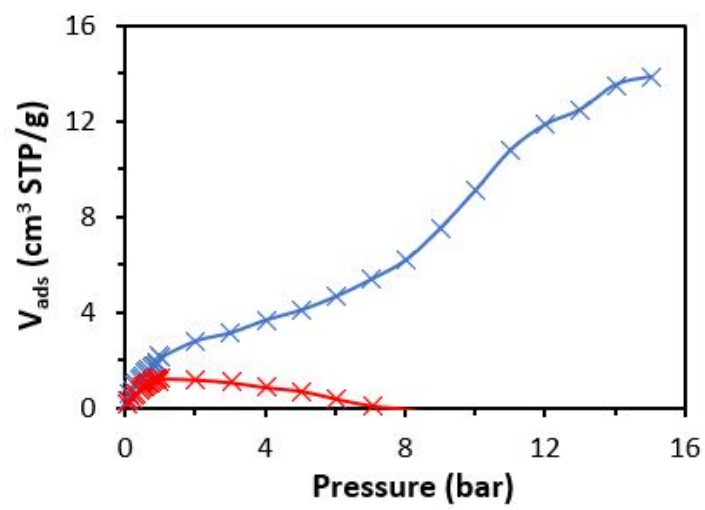

9 Figure 12. High-pressure ethylene (blue) and ethane (red) physisorption isotherms at $298 \mathrm{~K}$ of 10 3D-printed CPL-1 monoliths.

11 Ethane and ethylene isotherms were measured on 3D-printed CPL-1-based solids (Figure

12 12). Interestingly, no adsorption of ethane is observed above 8 bar, while adsorption of ethylene

13 increases with increasing pressure up to 16 bar following a type IV isotherm. ${ }^{46}$ Furthermore, the 14 3D printed solids did not show any visible defect after three adsorption/desorption cycles.

15 Thus, the robocasting technique is appropriate to shape MOF-based solids for high pressure 16 gas storage and separation, as long as the gravimetric capacity is the critical parameter. Little to 17 no effect of the presence of HEC and PVA is observed, beyond their contribution to the total solids 
1

2

3

4

5

6

7

8

9

10

11

12

13

14

15

16

17

18

19

20

21

22

23

24

25

26

27

28

29

30

31

32

33

34

35

36

37

38

39

40

41

42

43

44

45

46

47

48

49

50

51

52

53

54

55

56

57

58

59

60

1 weight. However, more investigation is required in order to densify the final solids and to increase

2 their volumetric capacity as discussed above, which is highly desirable for industrial applications. 


\section{CONCLUSION}

2 A modified 3D printer was used for the controlled deposition of inks, formulated from four

3 different MOF powders and in the presence of low proportions of a binder (2-hydroxyethyl

4 cellulose) and a plasticizer (polyvinyl alcohol). Owing to the determination of optimal shear-

5 thinning properties, a series of MOF-based solids with controlled macroscale morphology were

6 fabricated. All characterization techniques indicate that the 3D printing process impacted only

7 slightly the MOFs' textural and structural properties. Indeed, the solids displayed a permanent

8 microporosity that is comparable to the initial powders. Interestingly, SEM analysis highlighted

9 that the 3D-printed solids present a high degree of voids, due to the fast drying step employed.

10 Nonetheless, the solids present high compressive strength, only one to two orders of magnitude

11 lower than dense binderless pellets. When used for high-pressure methane storage (HKUST-1,

12 ZIF-8, and UiO-66-NH ${ }_{2}$ ), or for ethane/ethylene separation (CPL-1), the 3D-printed solids

13 displayed performances which are in line with the literature. This preliminary work needs more

14 investigation, especially for densifying the 3D-printed solids and improving their mechanical

15 robustness, but also for studying their stability in typical process conditions over cycling.

16 Especially, the 3D printer should be further modified to allow the processing of highly viscous

17 inks with shear-thinning properties, about one order of magnitude higher than the inks presented

18 herein. However, the authors believe that this study paves the way to the preparation of highly

19 porous MOF-based solids with designs completely adaptable to the applications: microreactors,

20 adsorbent beds, or separation membranes with specific morphologies to name a few. 


\section{ASSOCIATED CONTENT}

2 Supporting Information. The Supporting Information is available free of charge on the ACS

3 Publications website at DOI: .

4 Additional characterization include photographs, FT-IR spectra, SEM micrographs, viscosity

5 curves, $\mathrm{H}_{2} \mathrm{O}$ and $\mathrm{N}_{2}$ isotherms. The following files are available free of charge.

\section{AUTHOR INFORMATION}

\section{Corresponding Authors}

8 *Mail: jeremy.dhainaut@univ-lille.fr(J.D.); shuhei.furukawa@icems.kyoto-u.ac.jp (S.F.).

\section{Present Address}

$10 †$ Univ. Lille, CNRS, Centrale Lille, Univ. Artois, UMR 8181 - UCCS - Unité de Catalyse et

11 Chimie du Solide, F-59000 Lille, France.

\section{Author Contributions}

13 J.D and S.F. conceived and designed the project. J.D. prepared the HKUST-1 and UiO-66-NH 14 powders, the 3D-printed solids, and the pellets, and did all characterizations. M.B. prepared the 15 CPL-1 powders and did all high-pressure gas sorption experiments. J.D., U.R. and K.K. did all 16 mechanical tests. J.D. M.B. and S.F. analyzed the data and wrote the manuscript. All authors 17 discussed the results and commented on the manuscript.

18 ACKNOWLEDGMENT 
1 J.D. is grateful to the JSPS Postdoctoral Fellowship for Foreign Researchers (grant number

2 NoPE18023). The authors thank the iCeMS Analysis Center for access to analytical

3 instruments.

\section{REFERENCES}

5 (1) Yaghi, O. M.; O’Keeffe, M.; Ockwig, N. W.; Chae, H. K.; Eddaoudi, M.; Kim, J. Reticular 6 Synthesis and the Design of New Materials. Nature 2003, 423, 705-714.

7 (2) Kitagawa, S.; Kitaura, R.; Noro, S.-I. Functional Porous Coordination Polymers. Angew. Chem. 8 Int. Ed. 2004, 43, 2334-2375.

9 (3) Mueller, U.; Schubert, M.; Teich, F.; Puetter, H.; Schierle-Arndt, K.; Pastré, J. Metal-Organic 10 Frameworks-Prospective Industrial Applications. J. Mater. Chem. 2006, 16, 626-636.

11 (4) Mason, J. A.; Veenstra, M.; Long, J. R. Evaluating Metal-Organic Frameworks for Natural Gas 12 Storage. Chem. Sci. 2014, 5, 32-51.

13 (5) Suh, M. P.; Park, H. J.; Prasad, T. K.; Lim, D.-W. Hydrogen Storage in Metal-Organic 14 Frameworks. Chem. Rev. 2012, 112, 782-835.

15 (6) Qiu, S.; Xue, M.; Zhu, G. Metal-Organic Framework Membranes: From Synthesis to 16 Separation Application. Chem. Soc. Rev. 2014, 43, 6116-6140.

17 (7) Rubio-Martinez, M.; Hadley, T. D.; Batten, M. P.; Constanti-Carey, K.; Barton, T.; Marley, 18 D.; Mönch, A.; Lim, K.-S.; Hill, M. R. Scalability of Continuous Flow Production of Metal19 Organic Frameworks. ChemSusChem 2016, 9, 938-941.

20 (8) Carné-Sánchez, A.; Imaz, I.; Cano-Sarabia, M.; Maspoch, D. A Spray-Drying Strategy for 21 Synthesis of Nanoscale Metal-Organic Frameworks and their Assembly into Hollow 22 Superstructures. Nature Chem. 2013, 5, 203-211.

23 (9) Beldon, P. J.; Fábián, L.; Stein, R. S.; Thirumurugan, A.; Cheetham, A. K.; Friščić, T. Rapid 
1 Room-Temperature Synthesis of Zeolitic Imidazolate Frameworks by using Mechanochemistry.

2 Angew. Chem. Int. Ed. 2010, 49, 9640-9643.

3 (10) Doménech, A.; García, H.; Doménech-Carbó, M. T.; Llabrés-i-Xamena, F. Electrochemistry

4 of Metal-Organic Frameworks: A Description from Voltammetry of Microparticles Approach. $J$.

5 Phys. Chem. C 2007, 111, 13701-13711.

6 (11) Editorial, Nature Chem. 2016, 8, 987.

7 (12) Wu, D.; Zhou, J.; Li, Y. Mechanical Strength of Solid Catalysts: Recent Developments and

8 Future Prospects. AIChE J. 2007, 53, 2618-2629.

9 (13) Dhainaut, J.; Avci-Camur, C.; Troyano, J.; Legrand, A.; Canivet, J.; Imaz, I.; Maspoch, D.;

10 Reinsch, H.; Farrusseng, D. Systematic Study of the Impact of MOF Densification into Tablets on

11 Textural and Mechanical Properties. CrystEngComm 2017, 19, 4211-4218.

12 (14) Valekar, A. H.; Cho, K.-H.; Lee, U.-H.; Lee, J. S.; Yoon, J. W.; Hwang, Y. K.; Lee, S. G.;

13 Cho, S. J.; Chang, J.-S. Shaping of Porous Metal-Organic Framework Granules using Mesoporous $14 \rho$-Alumina as a Binder. RSC Adv. 2017, 7, 55767-55777.

15 (15) Spjelkavik, A. I.; Aarti; Divekar, S.; Didriksen, T.; Blom, R. Forming MOFs into Spheres by 16 Use of Molecular Gastronomy Methods. Chem. Eur. J. 2014, 20, 8973-8978.

17 (16) Tian, T.; Zeng, Z.; Vulpe, D.; Casco, M. E.; Divitini, G.; Midgley, P. A.; Silvestre-Albero, J.;

18 Tan, J.-C.; Moghadam, P. Z.; Fairen-Jimenez, D. A Sol-Gel Monolithic Metal-Organic Framework 19 with Enhanced Methane Uptake. Nature Mater. 2018, 17, 174-179.

20 (17) Fu, Q.; Wen, L.; Zhang, L.; Chen, X.; Pun, D.; Ahmed, A.; Yang, Y.; Zhang, H. Preparation 21 of Ice-Templated MOF-Polymer Composite Monoliths and their Application for Wastewater 22 Treatment with High Capacity and Easy Recycling. ACS Appl. Mater. Interfaces 2017, 9, 33979$23 \quad 33988$. 
1 (18) Hara, Y.; Kanamori, K.; Nakanishi, K. Self-Assembly of Metal-Organic Frameworks into

2 Monolithic Materials with Highly Controlled Trimodal Pore Structures. Angew. Chem. Int. Ed. $3 \mathbf{2 0 1 9}, 58,19047-19053$.

4 (19) Stassen, I.; Styles, M.; Grenci, G.; Van Gorp, H.; Vanderlinden, W.; De Feyter, S.; Falcaro, 5 P.; De Vos, D.; Vereecken, P.; Ameloot, R. Chemical Vapour Deposition of Zeolitic Imidazolate 6 Framework Thin Films. Nature Mater. 2016, 15, 304-310.

7 (20) Chen, Z.; Li, Z.; Li, J.; Liu, C.; Lao, C.; Fu, Y.; Liu, C.; Li, Y.; Wang, P.; He, Y. 3D Printing 8 of Ceramics: A Review. J. Eur. Ceram. Soc. 2019, 39, 661-687.

9 (21) Kreider, M. C.; Sefa, M.; Fedchak, J. A.; Scherschligt, J.; Bible, M.; Natarajan, B.; Klimov, 10 N. N.; Miller, A. E.; Ahmed, Z.; Hartings, M. R. Toward 3D Printed Hydrogen Storage Materials 11 Made with ABS-MOF Composites. Polym. Adv. Technol. 2018, 29, 867-873.

12 (22) Evans, K. A.; Kennedy, Z. C.; Arey, B. W.; Christ, J. F.; Schaef, H. T.; Nune, S. K.; Erikson, 13 R. L. Chemically Active, Porous 3D-Printed Thermoplastic Composites. ACS Appl. Mater. 14 Interfaces 2018, 10, 15112-15121.

15 (23) Halevi, O.; Tan, J. M. R.; Lee, P. S.; Magdassi, S. Hydrolytically Stable MOF in 3D-Printed 16 Structures. Adv. Sustainable Syst. 2018, 2, 1700150.

17 (24) Thakkar, H.; Eastman, S.; Al-Naddaf, Q.; Rownaghi, A. A.; Rezaei, F. 3D-Printed Metal18 Organic Framework Monoliths for Gas Adsorption Processes, ACS Appl. Mater. Interfaces 2017, $199,35908-35916$.

20 (25) Sultan, S.; Abdelhamid, H. N.; Zou, X.; Mathew, A. P. CelloMOF: Nanocellulose Enabled 21 3D Printing of Metal-Organic Frameworks. Adv. Funct. Mater. 2019, 29, 1805372.

22 (26) Young, A. J.; Guillet-Nicolas, R.; Marshall, E. S.; Kleitz, F.; Goodhand, A. J.; Glanville, L. 23 B. L.; Reithofer, M. R.; Chin, J. M. Direct Ink Writing of Catalytically Active UiO-66 Polymer 
1 Composites. Chem. Commun. 2019, 55, 2190-2193.

2 (27) Bonneau, M.; Lavenn, C.; Ginet, P.; Otake, K.-I.; Kitagawa, S. Upscale Synthesis of a Binary

3 Pillared Layered MOF for Hydrocarbon Gas Storage and Separation. Green Chem. 2020, Advance

4 Article.

5 (28) Katz, M. J.; Brown, Z. J.; Colón, Y. J.; Siu, P. W.; Scheidt, K. A.; Snurr, R. Q.; Hupp, J. T.;

6 Farha, O. K. A Facile Synthesis of UiO-66, UiO-67 and their Derivatives. Chem. Commun. 2013, $7 \quad 49,9449-9451$.

8 (29) Huo, J.; Brightwell, M.; El Hankari, S.; Garai, A.; Bradshaw, D. A Versatile, Industrially

9 Relevant, Aqueous Room Temperature Synthesis of HKUST-1 with High Space-Time Yield. J.

10 Mater. Chem. A 2013, 1, 15220-15223.

11 (30) Couck, S.; Cousin-Saint-Remi, J.; Van der Perre, S.; Baron, G. V.; Minas, C.; Ruch, P.;

12 Denayer, J. F. M. 3D-Printed SAPO-34 Monoliths for Gas Separation, Micro. Meso. Mater. 2018, $13 \quad 255,185-191$.

14 (31) Lefevere, J.; Protasova, L.; Mullens, S.; Meynen, V. 3D-Printing of Hierarchical Porous ZSM15 5: The Importance of the Binder System. Mater. Design 2017, 134, 331-341.

16 (32) Lewis, T. B.; Nielsen, L. E. Viscosity of Dispersed and Aggregated Suspensions of Spheres.

17 Trans. Soc. Rheol. 1968, 12, 421-443.

18 (33) Baek, G.; Kim, C. Rheological Properties of Carbopol Containing Nanoparticles. J. Rheol. 19 2011, 55, 313-330.

20 (34) Pabst, W.; Gregorová, E.; Bertold, C. Particle Shape and Suspension Rheology of Short-Fiber 21 Systems. J. Eur. Ceram. Soc. 2006, 26, 149-160.

22 (35) Kandiah, M.; Nilsen, M. H.; Usseglio, S.; Jakobsen, S.; Olsbye, U.; Tilset, M.; Larabi, C.; 23 Quadrelli, E. A.; Bonino, F.; Lillerud, K. P. Synthesis and Stability of Tagged UiO-66 Zr-MOFs. 
1 Chem. Mater. 2010, 22, 6632-6640.

2 (36) Todaro, M.; Buscarino, G.; Sciortino, L.; Alessi, A.; Messina, F.; Taddei, M.; Ranocchiari, 3 M.; Cannas, M.; Gelardi, F.M. Decomposition Process of Carboxylate MOF HKUST-1 Unveiled 4 at the Atomic Scale Level. J. Phys. Chem. C 2016, 120, 12879-12889.

5 (37) Horike, S.; Shimomura, S.; Kitagawa, S. Soft Porous Crystals. Nature Chem. 2009, 1, 6956704.

7 (38) Vehring, R. Pharmaceutical Particle Engineering via Spray Drying, Pharm. Res. 2008, 25, $8 \quad 999-1022$.

9 (39) Studart, A. R.; Gonzenbach, U. T.; Tervoort, E.; Gauckler, L. J. Processing Routes to 10 Microporous Ceramics: A Review. J. Am. Ceram. Soc. 2006, 89, 1771-1789.

11 (40) Policicchio, A.; Filosa, R.; Abate, S.; Desiderio, G.; Colavita, E. Activated Carbon and Metal 12 Organic Framework as Adsorbent for Low-Pressure Methane Storage Applications: An Overview. 13 J. Porous Mater. 2017, 24, 905-922.

14 (41) He, Y.; Zhou, W.; Qian, G.; Chen, B. Methane Storage in Metal-Organic Frameworks. Chem. 15 Soc. Rev. 2014, 43, 5657-5678.

16 (42) Mu, L.; Liu, B.; Liu, H.; Yang, Y.; Sun, C.; Chen, G. A Novel Method to Improve the Gas 17 Storage Capacity of ZIF-8. J. Mater. Chem. 2012, 22, 12246-12252.

18 (43) Cmarik, G. E.; Kim, M.; Cohen, S. M.; Walton, K. S. Tuning the Adsorption Properties of 19 UiO-66 via Ligand Functionalization. Langmuir 2012, 28, 15606-15613.

20 (44) Kitaura, R.; Kitagawa, S.; Kubota, Y.; Kobayashi, T. C.; Kindo, K.; Mita, Y.; Matsuo, A.; 21 Kobayashi, M.; Chang, H.-C.; Ozawa, T. C.; Suzuki, M.; Sakata, M.; Takata, M. Formation of a 22 One-Dimensional Array of Oxygen in a Microporous Metal-Organic Solid. Science 2002, 298, $23 \quad 2358-2361$. 
1 (45) Kishida, K.; Watanabe, Y.; Horike, S.; Watanabe, Y.; Okumura, Y.; Hijikata, Y.; Sakaki, S.;

2 Kitagawa, S. DRIFT and Theoretical Studies of Ethylene/Ethane Separation on Flexible and

3 Microporous $\left[\mathrm{Cu}_{2}(2,3-\text { pyrazinedicarboxylate })_{2} \text { (pyrazine) }\right]_{\mathrm{n}}$. Eur. J. Inorg. Chem. 2014, 17, $2747-$ 42752.

5 (46) Zheng, F.; Guo, L.; Gao, B.; Li, L.; Zhang, Z.; Yang, Q.; Yang, Y.; Su, B.; Ren, Q.; Bao, Z. 6 Engineering the Pore Size of Pillared-Layer Coordination Polymers Enables Highly Efficient 7 Adsorption Separation of Acetylene from Ethylene. ACS Appl. Mater. Interfaces 2019, 11, $28197-$ 828204.

9 (47) Li, L.; Lin, R.-B.; Krishna, R.; Li, H.; Xiang, S.; Wu, H.; Li, J.; Zhou, W.; Chen, B. 10 Ethane/Ethylene Separation in a Metal-Organic Framework with Iron-Peroxo Sites. Science 2018, $11362,443-446$.

12 